\title{
Simulation par ordinateur de la ligne Haute Tension en régime permanent dans les conditions aléatoires
}

\author{
S. M. Debbal \\ Department of electronic, Faculty of engineering science, university Aboubekr Belkaid, \\ Tlemcen, BP 119, Tlemcen 13000, Algeria \\ (Reçu le 24 Janvier 2005, accepté le 06 Juin 2005) \\ * Correspondance et tirés à part, e-mail : adebbal@yahoo.fr

\section{Résumé} \\ Ce travail consiste à la simulation par ordinateur du fonctionnement de la ligne Haute \\ Tension (HT) et du réseau de distribution HT. Ceci s'est fait par l'établissement d'un \\ modèle pouvant tenir compte de la distribution de l'énergie entre les différents éléments \\ du réseau HT dans des conditions normales et aléatoires de fonctionnement. Les \\ résultats ainsi obtenus permettent de comprendre et d'agir sur le fonctionnement du \\ réseau dans le but d'une amélioration et d'une optimisation de la distribution de \\ l'énergie.
}

Mots-clés : Ligne haute tension (HT), Réseau HI, distribution, modélisation, simulation, optimisation, condition normale, condition aléatoire.

\section{Abstract \\ Computing simulation of the high voltage line operation during permanent state in random conditions}

The aim of this work consists of the simulation by computer for the operation of the High Voltage (HV) line and also of the network distribution. This will be done by the establishment of a model which can take account of the energy distribution between the various elements of the HV network under normal and random operation conditions. The results we obtain make it possible to understand and act upon the network operation. This allows an improvement and an optimisation of the energy distribution.

Keywords: High Voltage line (HV), HV network, distribution, modeling, simulation, normal condition, random condition. 


\section{Introduction.}

L'étude des lignes HT a toujours fait l'objet d'un grand intérêt de la part des Chercheurs. C'est parfaitement compréhensible si on constate son importance dans la gestion économique de la production électrique et du rôle traditionnel de transport et de distribution visant à assurer une alimentation électrique convenable et sécurisée à la clientèle.

La planification du réseau de grand transport doit être à même de réaliser le compromis optimal entre le cô̂t de l'optimisation du réseau et les gains qu'ils permettent, tant en matière de dépenses de combustibles qu'en amélioration de la qualité du service. Malheureusement, les problèmes à résoudre sont trop complexes; il faut donc procéder par approximations et itérations successives en manipulant des sous problèmes plus simples.

En 1975, J.C Dodu [1] s'est proposé de résoudre le problème du modèle dynamique d'optimisation à long terme d'un réseau de transport d'énergie électrique de la façon suivante : connaissant l'évolution de la demande, la composition et la localisation du parc de production, il peut déterminer les capacités (puissances) de transit des liaisons du réseau aux différents instants de la période d'étude de telle sorte que la somme actualisée des coûts d'investissements, d'exploitation et défaillance soit minimale. (l'évolution de la demande est donnée sous la forme de courbes ou monotones de charges annuelles; chaque monotone comportant elle-même plusieurs moments : heure de pointe, heure pleine, heure creuse) [2]. Le réseau de distribution sera représenté par un schéma faisant ressortir le nombre des sommets (ou nœuds) et des lignes HT.

En 1980, Arnaud Hertz et al. [3] ont mis au point un programme appelé ॥ CORALI ॥ qui est un programme de calcul pour la planification et une aide à la conduite des réseaux électriques moyenne tension d'électricité. Pour ce faire, il effectue une recherche automatique du meilleur schéma d'exploitation possible compte tenu des différents impératifs de la distribution ou desserte.

Toujours en 1980, Szczesny Kujsczyk [4] trouve un système informatique qui est utilisé pour l'exploitation des réseaux de distribution à moyenne tension. II permet aussi de déterminer les points de bouclages optimaux du point de vue de la réduction des pertes.

En 1982, Sozanki Niezowodnosc [5] explique d'une façon détaillée l'importance des monotones de la demande sur le fonctionnement du réseau HT. II donne en plus un aperçu sur le phénomène des défaillances au niveau des lignes HT. 


\section{Description du problème}

Compte tenu de l'étendue du problème et dans l'état actuel des moyens de calcul, l'étude du développement à long terme d'un réseau de transport d'énergie électrique au moyen d'un modèle mathématique utilisant les méthodes de la recherche opérationnelle ne peut se faire qu'au prix d'une représentation très schématisée du réseau et moyennant l'adoption d'un certain nombre d'hypothèses simplificatrices assez larges. Nous avons retenu les approximations suivantes pour le modèle que nous proposons [2] :

- La circulation de l'énergie dans le réseau obéit à la première loi de Kirchhoff.

- Les demandes d'énergie dans les divers postes ou récepteurs suivent des monotones aléatoires ajustés sur les courbes des besoins en puissance.

- La fiabilité du réseau est considérée comme un phénomène aléatoire agissant directement sur la puissance nécessaire du réseau.

II est clair qu'un tel modèle ne peut prétendre résoudre le problème du développement à très long terme d'un réseau de transport d'énergie dans toute sa complexité ; néanmoins l'intérêt et l'ambition de cette étude est :

-D'étudier comment varient la structure et le dimensionnement du réseau en fonction des paramètres fondamentaux propres au réseau tels que la demande de l'énergie et son évolution ainsi que sa fiabilité.

-D'obtenir des résultats assez satisfaisants dans l'ensemble qui nous permettront de prendre une décision pour l'amélioration du réseau ou son optimisation.

\section{Modèles de simulation proposés}

II n'existe pas, et n'existera probablement jamais d'approche totalement intégrée de la planification des grands réseaux de transport et d'interconnexion. Les problèmes à résoudre sont trop complexes. II faut donc faire un découpage et une hiérarchisation des sous-problèmes extrêmement diversifiés. Les modèles de simulation proposés s'inscrivent ainsi dans cette optique.

\section{3-1. Modèle de simulation de la ligne Haute Tension (HT) seule $[6,7]$}

Pour le modèle de simulation de la ligne HT seule il suffit de considérer le schéma électrique présenté à la Figure 1. 


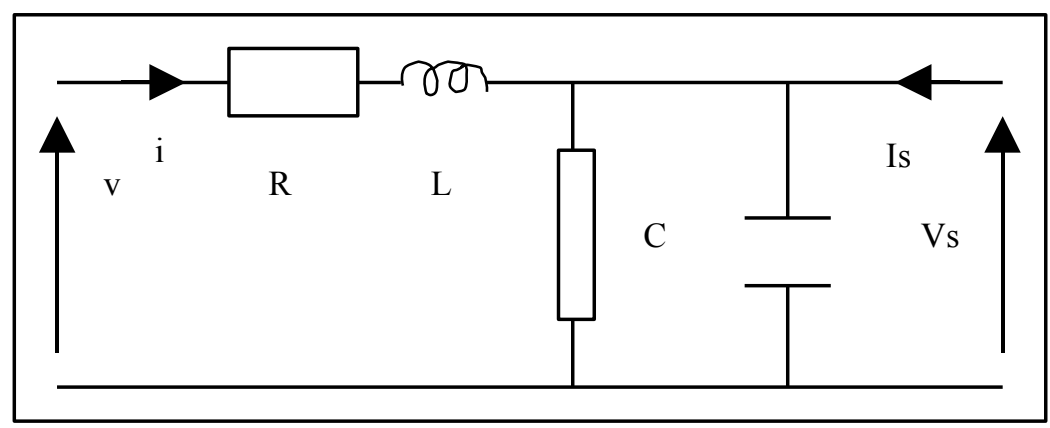

Figure 1 : Schéma électrique équivalent de la ligne HT.

$R$ : résistance linéique de la ligne HT.

$G$ : conductance linéique de la ligne HT.

$C$ : capacité linéique de la ligne HT.

$L$ : inductance linéique de la ligne $H T$.

$d x$ : élément de longueur de la ligne HT.

Ces paramètres sont reliés entre- eux par les équations différentielles suivantes $[6,7]$ :

$\frac{\partial^{2} v}{\partial x^{2}}=L C \frac{\partial^{2} v}{\partial t^{2}}+(R C+L G) \frac{\partial v}{\partial t}+R G v$

$\frac{\partial^{2} i}{\partial x^{2}}=L C \frac{\partial^{2} i}{\partial t^{2}}+(R C+L G) \frac{\partial i}{\partial t}+R G i$

En pratique, on néglige très souvent la valeur numérique de la conductance $(G \approx 0)$. Cette approximation facilite considérablement les calculs.

\section{3-2. Modèle de simulation du tronçon transformateur-récepteur $[6,7]$}

Les paramètres importants qui agissent au niveau de ce modèle sont donnés par la Figure 2 ; ils sont :
$\underline{R}$ : récepteur
$\underline{G}:$ générateur.
I : transformateur
$C$ : capacité linéique de la ligne HT
$L$ : inductance linéique de la ligne HT
$R$ : résistance linéique de la ligne HT 


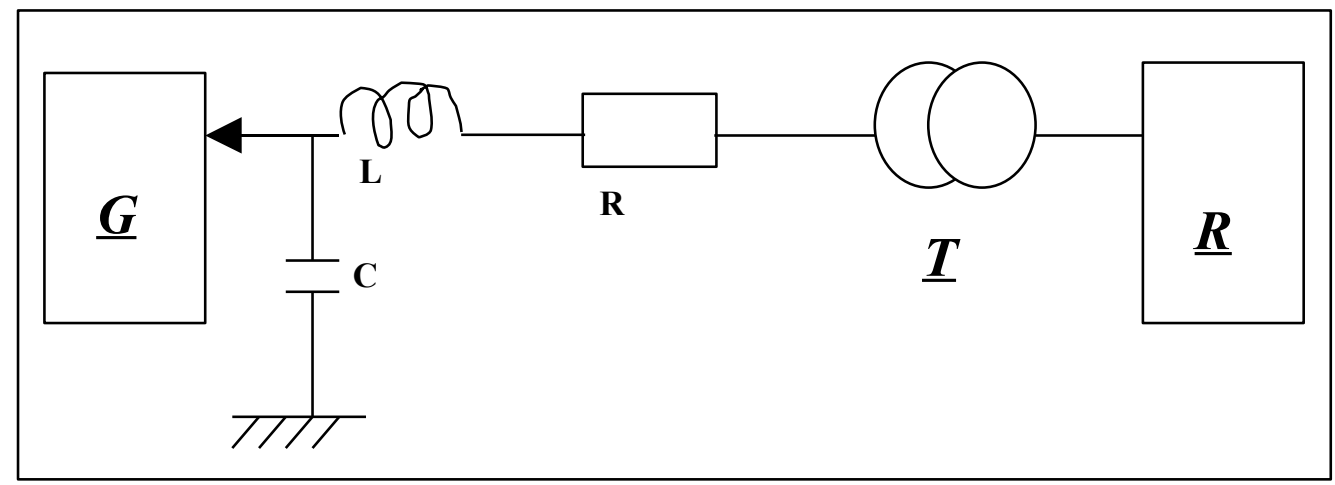

Figure 2 : Schéma électrique équivalent du tronçon transformateur-récepteur

Pour faire apparaître le concept de puissance à ce niveau nous allons utiliser directement le schéma de la Figure 3. Sur cette figure, nous remarquons que le transformateur est au carrefour entre la ligne HT (qui vient du générateur ou d'un nœud du réseau HT) et la ligne moyenne tension (MT). Les puissances concernées dans cette configuration sont les suivantes:

- $P$ : puissance nécessaire à l'entrée du transformateur pour alimenter le récepteur.

- $P I$ : puissance perdue par le transformateur.

- P2 : puissance utilisée par le transformateur (au bénéfice du récepteur $R$ ).

D'où la figure suivante :

HT

MT

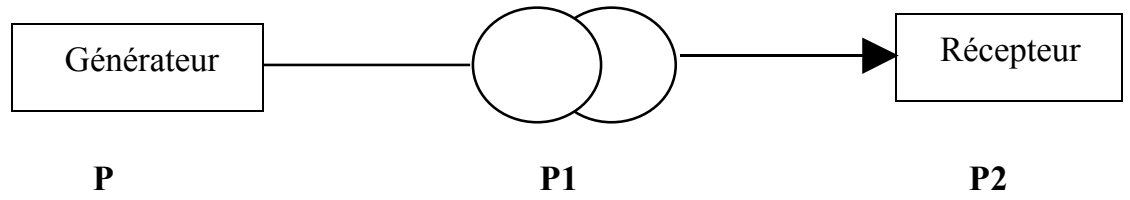

Figure 3 : Schéma de puissance du tronçon transformateur-récepteur

Connaissant les besoins en puissance au niveau du récepteur (P2) et la puissance perdue par le transformateur $(\mathrm{PI})$, il est facile de connaître la puissance $(\mathrm{P})$ nécessaire à appliquer à l'entrée du transformateur, par le principe de la conservation d'énergie :

$$
\mathrm{P}=\mathrm{P} 1+\mathrm{P} 2
$$




\section{3-3. Modèle de simulation de la ligne HT et fiabilité}

Durant son fonctionnement la ligne HT est susceptible d'être exposée à des défaillances et à des réparations. Ce phénomène fait l'objet d'étude du paramètre de fiabilité de la ligne HT [8-10]

La fiabilité permet donc de donner des indications très appréciables sur l'état de fonctionnement (très souvent aléatoire) des éléments d'un système.

Le modèle de fiabilité que nous pouvons proposer pour un seul élément de la ligne est représenté par la Figure 4.

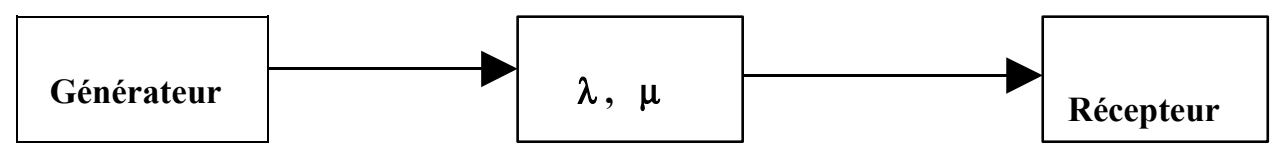

Figure 4 : Paramètres de fiabilité pour un seul élément de la ligne HT Avec:

$\lambda$ : taux de défaillance

$\mu$ : taux de réparation.

Comme dans cette étude nous avons considéré que la ligne est composée de deux éléments (la ligne HT et le transformateur) il est donc nécessaire de rechercher la fiabilité de l'ensemble et son modèle. La configuration de l'ensemble sera une configuration série car la défaillance d'un élément entraînera systématiquement l'arrêt du fonctionnement de toute la ligne HT. Le modèle de fiabilité proposé est présenté à la Figure 5.

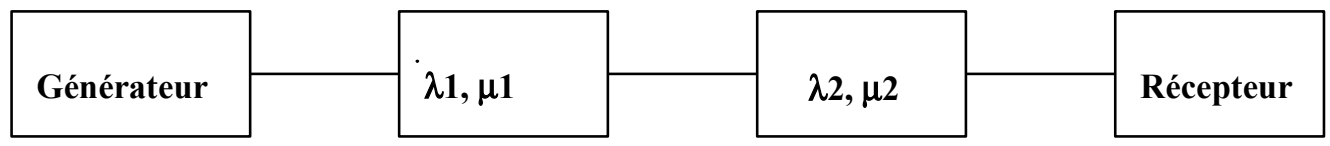

Figure 5 : Modèle de fiabilité du tronçon ligne HT et transformateur

$\lambda$ I: taux de défaillance de la ligne HT seule

$\mu l$ : taux de réparation de la ligne $H T$ seule

$\lambda 2$ : taux de défaillance du transformateur

$\mu 2$ : taux de réparation du transformateur

La fiabilité $R$ en fonction du temps $t$ des éléments respectifs est donc : 
- Pour la ligne HT seule :

$R_{1}(t)=\frac{\mu_{1}}{\lambda_{1}+\mu_{1}}+\frac{\lambda_{1}}{\lambda_{1}+\mu_{1}} \cdot \exp \left\{-\left(\mu_{1}+\lambda_{1}\right) t\right\}$

*Pour le transformateur :

$R_{2}(t)=\frac{\mu_{2}}{\lambda_{2}+\mu_{2}}+\frac{\lambda_{2}}{\lambda_{2}+\mu_{2}} \cdot \exp \left\{-\left(\mu_{2}+\lambda_{2}\right) t\right\}$

*Pour la ligne HT + transformateur :

$$
R(t)=R_{1}(t)+R_{2}(t)
$$

Le diagramme de la Figure 6 donne un aperçu de la relation temporelle entre les différents taux $\left(\lambda_{i}, \mu_{i}\right)$ :

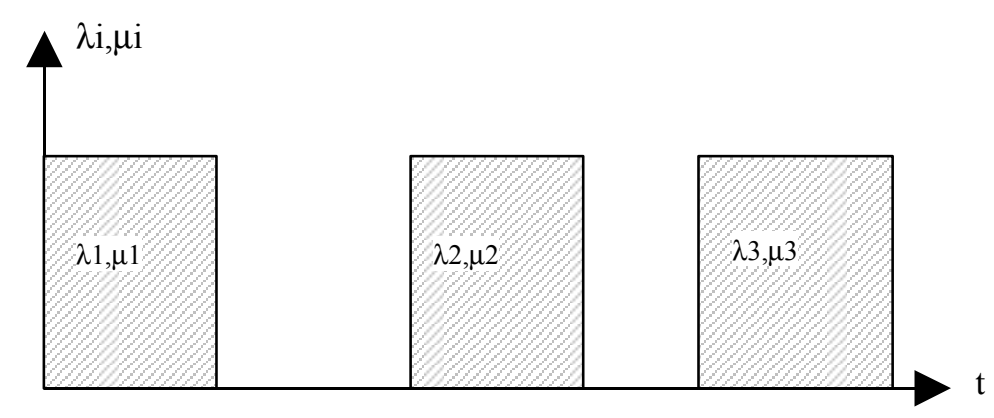

Figure 6 : Exemple de fonctionnement d'une ligne HT avec trois défaillances et leurs réparations

Les zones hachurées correspondent aux intervalles de temps des pannes et de leurs réparations.

\section{3-4. Modèle de simulation du réseau de distribution HT}

Le réseau de distribution (HT) ne sera en fait qu'un assemblage de tous les modèles proposés ci-avant. Pour chaque nœud, nous aurons ainsi une configuration qui fera appel à l'association des trois modèles décrits antérieurement [11]. 


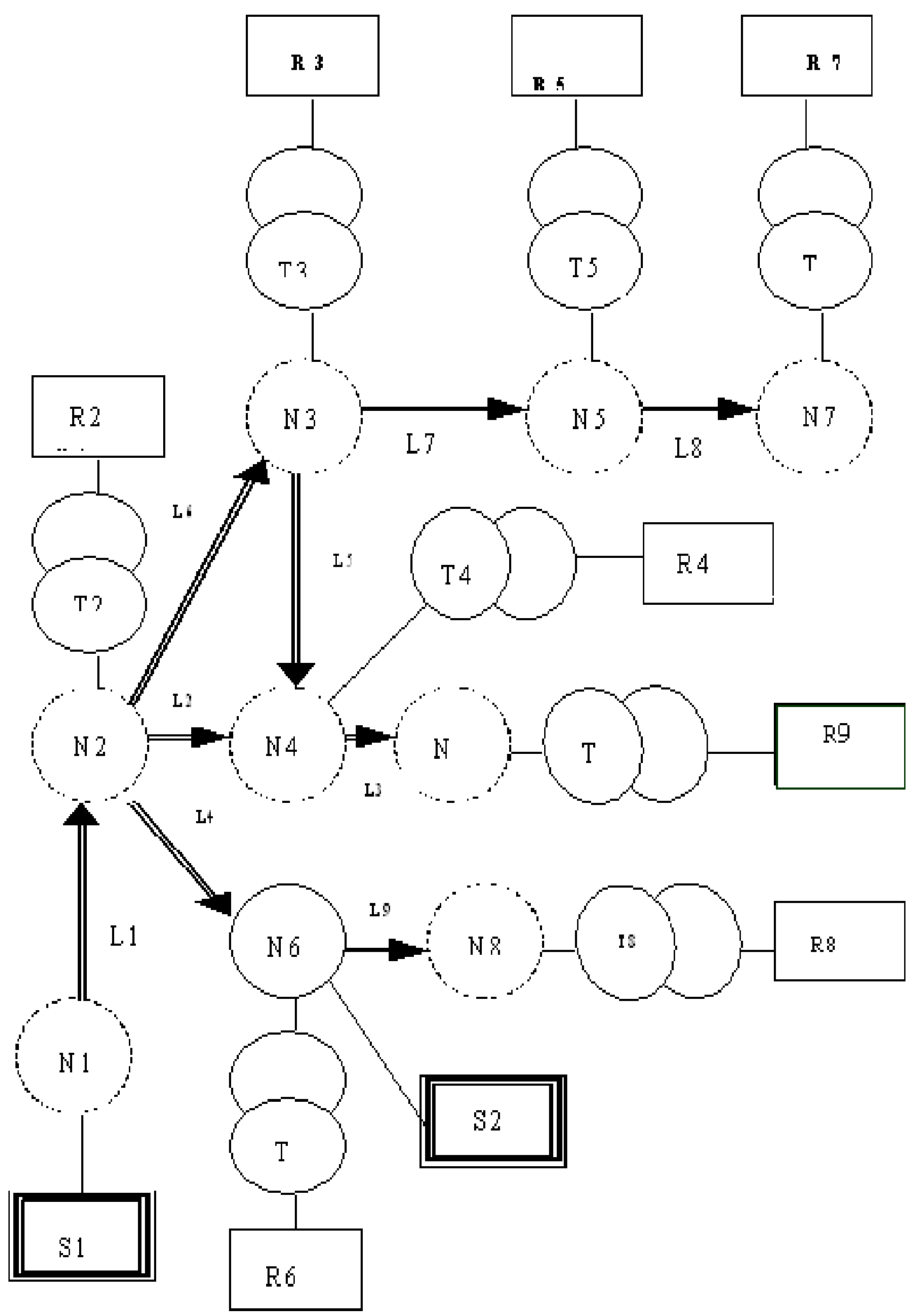

Figure 7 : Schéma du réseau de distribution $H T$ de la région Ouest de l'Algérie 
En outre, pour avoir un impact plus direct sur le monde de l'industrie, nous avons appliqué la simulation au cas réel d'un réseau de distribution de la région Ouest de l'Algérie. La Figure 7 présente le schéma de ce réseau, avec:

- SI-S2 : Générateurs ou sources HT

- T2-19: Transformateurs

- R2-R9 : Récepteurs

- LI-L9 : Lignes HT

-NI-N9 : Nauds d'interconnexion des lignes HT.

La puissance globale $\left(P_{s}\right)$ nécessaire au bon fonctionnement du réseau HT est considérée comme étant la somme des puissances injectées à l'entrée des différents transformateurs $\left(P_{i}\right)$.

$$
P_{s}=\sum_{i=1}^{N} P_{i}
$$

\section{Résultats et discussion}

\section{4-1. Paramètres d'entrée}

Les données à rentrer à l'ordinateur concernent :

- Les valeurs linéiques de la ligne $H T(R, G, C$ et $L)$

- Les paramètres de fiabilité $\left(\lambda_{i}, \mu_{i}\right)$.

- Les paramètres du transformateur (puissance)

- Les monotones (courbes de répartition de l'énergie) annuelle, mensuelle, hebdomadaire et journalière.

- La configuration du réseau de distribution (nombre de nœuds, de lignes HT, de récepteurs, de transformateurs)

- Le schéma graphique.

\section{4-2. Organigramme de simulation proposé}

L'organigramme de simulation du fonctionnement de la distribution au niveau du réseau proposé est donné par la Figure 8. 


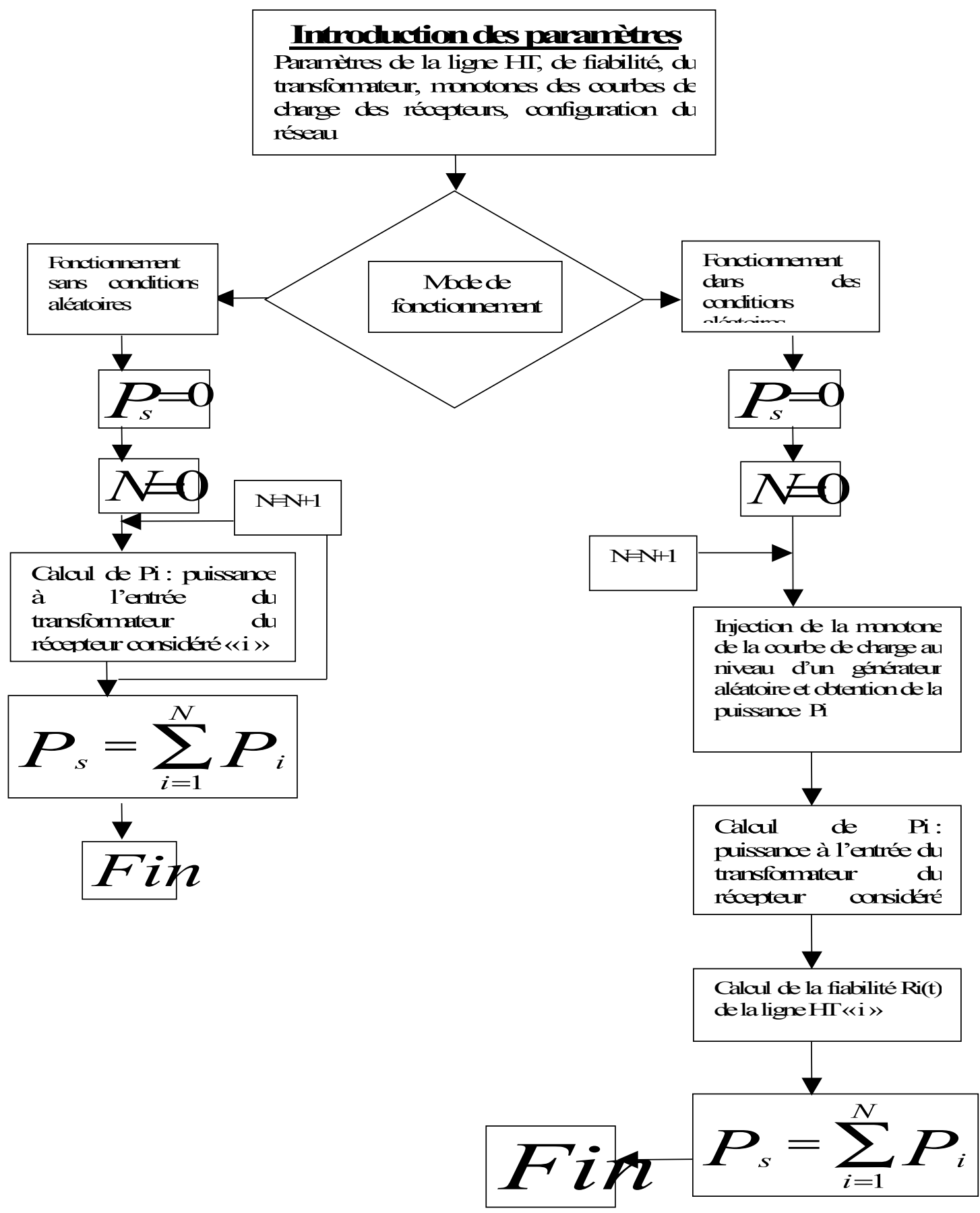

Figure 8: Organigramme du calcul de la puissance nécessaire au réseau pour les régimes aléatoire et non aléatoire 
$P_{s}$ étant la puissance nécessaire au réseau pour son fonctionnement normale on peut donc considérer deux cas :

- Le premier cas est celui du calcul de la puissance $P_{s}$ quand on néglige le côté aléatoire de la demande et de la fiabilité.

- Le deuxième cas est le calcul de la puissance $P_{s}$ lorsqu'on fait intervenir les deux phénomènes aléatoires de la demande (à travers le passage de la monotone par un générateur aléatoire) et de la fiabilité. La sortie du générateur aléatoire est ajustée sur l'allure de la demande en puissance . La sortie de ce générateur nous fournira donc une allure identique à celle de la demande en puissance mais avec des valeurs de puissances assez aléatoires pour simuler les évènements aléatoires du réseau. La variation pourra être ajustée selon le cas, suivant une loi exponentielle, normale, etc.

On calcule ainsi pour chaque nœud la puissance $P_{i}$ nécessaire (celle-ci est donnée par le besoin des récepteurs qui sont rattachés à ce nœud : monotones de charge) et on fait à la fin une sommation de toutes les puissances au niveau des nœuds pour obtenir en fin de compte la puissance nécessaire globale du réseau.

\section{4-3. Simulation}

Les résultats de la simulation en puissance du réseau électrique peuvent être présentés sous forme de tableaux renfermant toutes les valeurs des puissances mesurées et calculées. Cependant, pour une compréhension plus rapide du fonctionnement du réseau, les courbes de la Figure 9 paraissent être une approche plus efficace en fournissant de façon visible et très claire la différence recherchée entre les cas aléatoire et non aléatoire.

On peut donc noter que le principal résultat obtenu est l'élaboration et la mise au point d'un II simulateur " pouvant simuler le fonctionnement du réseau de distribution et suivre les différentes évolutions de la demande en puissance. La collecte de ces précieuses informations sera d'une grande utilité pour :

-déceler les anomalies de fonctionnement du réseau

-améliorer son état de fonctionnement

-optimiser son rendement.

Par ailleurs, on sait que les deux principales sources ou causes de phénomènes aléatoires sont la demande (ou la monotone de charge) et la fiabilité des lignes HT (on distinguera ici la fiabilité des lignes et celle du transformateur); en recueillant donc un nombre suffisant d'informations sur ces deux causes-là, l'estimation de la puissance nécessaire au bon fonctionnement du réseau de distribution sera alors assez rigoureuse. Le simulateur proposé tient tout de même compte de deux paramètres non négligeable du réseau à savoir la demande en puissance (monotone de charge) et la fiabilité 
(défaillance de la ligne et sa réparation). Ces deux paramètres ont donc un impact important sur la relation de la demande et de l'offre.

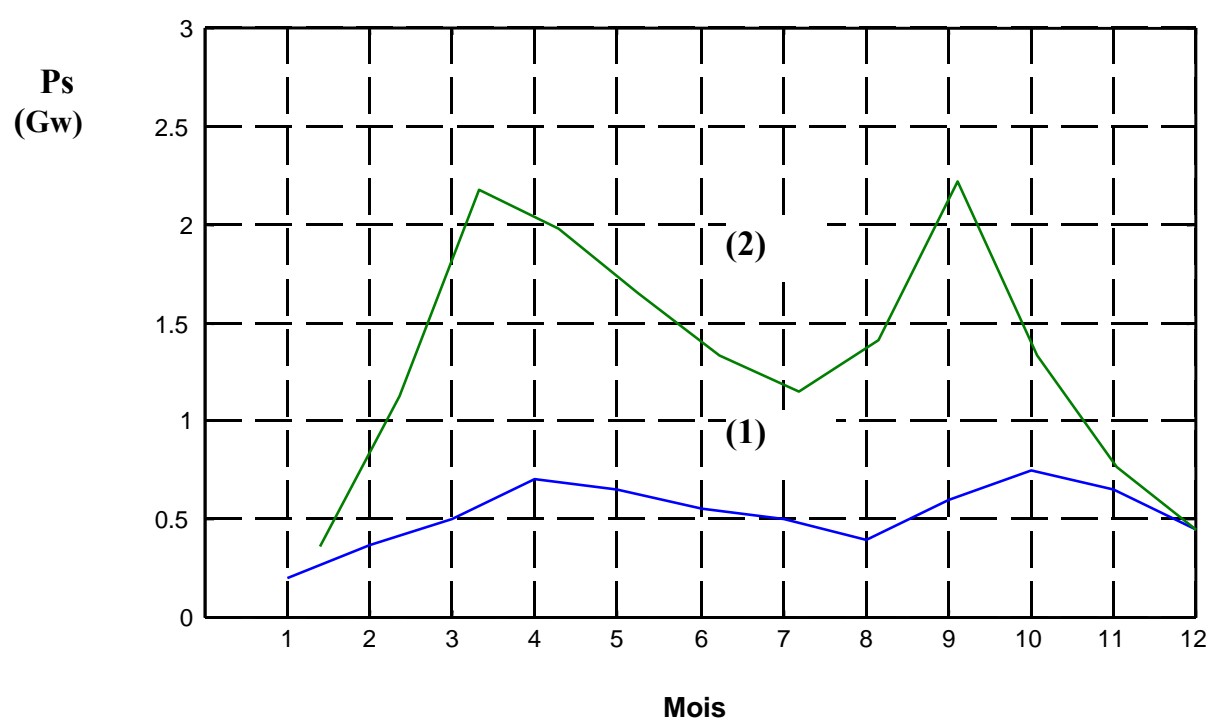

Figure 9 : Simulation de la puissance nécessaire au fonctionnement du réseau - en régime normale, courbe (1)

- en régime aléatoire, courbe (2)

Les résultats fournis par le simulateur sont donc présentés par deux courbes (Figure 9):

- la première, courbe de référence notéé (I) représente la puissance nécessaire au réseau dans des conditionnements de travail favorable (bonne fiabilité et demande régulière)

- la deuxième, courbe réelle notée (2) est tracée en fonction des paramètres pratiques et réels en faisant intervenir les phénomènes aléatoires qui sont dus à une mauvaise fiabilité et à une demande mal maîtrisée.

En superposant ces deux courbes c'est de faire coulisser la courbe réelle sur la courbe de référence en essayant de faire diminuer l'écart qui existe entre ces deux courbes. Ceci ne peut se faire qu'après plusieurs itérations d'optimisation du réseau en agissant sur les valeurs des éléments (ligne HT, transformateur, etc.). 


\section{CONCLUSION}

Au cours de ce travail, nous avons conçu et réalisé un "s simulateur " qui permet d'optimiser en puissance le fonctionnement d'un réseau électrique.

Ce simulateur peut être appliqué dans divers secteurs des services et industries de l'énergie électrique, notamment :

- Les bureaux d'étude pour une plus grande optimisation des réseaux de distribution afin de réduire au maximum le nombre de lignes HT ou la distance entre les divers éléments du réseau.

- Les services de maintenance pour déceler les diverses défaillances au niveau du réseau et prendre les mesures adéquates pour leur réparation.

- Les services de contrôle pour avoir un suivi régulier et continu de la relation offre et demande.

\section{Références}

[1] - J. C. DODU. II Modèle dynamique d'optimisation a long terme d'un réseau de transport d'énergie électrique II Bulletin de la direction des études et recherches, Electricité de France (EDF), série $C, n^{\circ} 2$ (1975), pp 5-16

[2] - F. COULON , M.JUFFER. IIntroduction à l'électrotechnique II. Traité d'électricité, volume l. Presses polytechniques et universitaires romandes. (1995) PP 41-81

[3] - A. HERTZ, D. GIRAUD et C. FAUST. II Un modèle pour la recherche de schéma d'exploitation sur les réseaux moyenne tension II, Revue générale de l'Electricité, $\mathrm{n}^{\circ} 2$ (1986) pp 133-148

[4] - S. KUJSCZYK. Système informatique pour la recherche du schéma d'exploitation optimal d'un réseau de distribution II, Revue générale de l'Electricité, n² 2 (1980) ppl49-152

[5] - S. NIEZOWODNOSC. "IEnergia elektryczng II. pwn warszowa. (1982) en polonais

[6] - G.I. ATABIEKOW. IITeoria liniowyek obwodw elektryczng II. pwn warszowa. (1967), en polonais

[7] - P. LAGNOTTE. ॥ Les installations électriques II. Edition Hermes Sciences Publications. (2000) PP 127-252

[8] - G. ZWINGELSTEIN. "La maintenance basée sur la fiabilité ॥. Guide pratique d'application de la RCM. (1996)

[9] - G. ZWINGELSTEIN. "Diagnostic des défaillances ॥. Théorie et pratique pour les systèmes industriels, (1995)

[10] - J.P PLANTARD. ॥ Fiabilité II, Polycopié de l'école Supérieure d'électricité de France (SUPELEC), (1981) pp 19-22

[11] - J. NEIRYNCK. "Théorie des réseaux de Kirchhoff ॥ . Traité d'électricité. Volume IV. Presses polytechniques et universitaires romandes, PP 109-146 